\title{
Ecological and epidemiological models are both useful for SARS-CoV-2
}

To the Editor - A longstanding debate exists on whether ecological phenomena should be modelled 'top-down' (modelling patterns that arise from mechanisms) or 'bottom-up' (modelling mechanisms to generate pattern). Recently, the discussion re-emerged in the context of modelling the spread of SARS-CoV-2 $2^{1}$. Simply put, the point made by Carlson et al. was that top-down correlative models are inappropriate, whereas

bottom-up epidemiological models are fine. Rather than opposing families of models, we argue that all have strengths and limitations and that judicious use of all available tools is the way forward.

\section{From process to pattern}

Regardless of the modelling approach used for infectious disease, one must start by understanding the mechanisms of transmission. A critical quantity is the reproduction number, $R_{0}$, which indicates how contagious the disease is, and which can be inferred by multiplication of the terms ${ }^{2}$ :

$$
R_{0}=D \times O \times T \times S
$$

where $D$ is the duration of the infectious period, $O$ represents the opportunities for infection, $T$ is the transmission probability and $S$ is the susceptibility.

For SARS-CoV-2, $D$ can be obtained by measuring the duration of infectiousness among infected individuals. Opportunities for infection, $O$, reflect the probability of human contact, which is affected by the density of humans and the magnitude and direction of movements. Transmission probability, $T$, reflects the likelihood of transmission given contact of a non-infected individual with an infected individual or surface. Finally, susceptibility to transmission, $S$, is dependent on the degree of immunity of the population, which is driven by previous exposure to SARS-CoV-2, other pathogens triggering immune responses to SARS-CoV- $2^{3}$, and the age structure of populations $s^{4}$.

Because the terms in the equation are multiplicative, if one term is brought near zero it will lead to sharp reductions in $R_{0}$. This underscores the importance of non-pharmaceutical approaches to manipulation of terms $O$ (for example, lock-downs and quarantines) or $T$ (for example, enforcement of behavioural rules).
Importantly, each term of the equation is driven by multiple parameters, and each one of them changes across space and time. So, $R_{0}$ is context-dependent. This makes it extremely difficult to parameterize epidemiological models with high accuracy. Although epidemiological models are based on known transmission mechanisms, parameters still need to be estimated empirically or experimentally, neither of which is without shortcomings. While empirical estimates suffer from the same noise affecting correlative models, experimentally derived values are constrained by simplifications of controlled experiments, which affect the realism of estimated values.

\section{From pattern to process}

A critical issue for top-down pattern analysis is whether variables of interest, like climate ${ }^{5}$ or concentration of pollutants ${ }^{6}$, have a mechanistic link to spread of the disease and how much that link affects transmission patterns. Using the DOTS equation and focusing on climate, we can speculate about the mechanistic links of SARS-CoV-2 and climate.

Firstly, it is unclear what the effects of climate might be on the duration, $D$, of the infectious period. However, it is not unusual that, under optimal climate conditions, immunological responses to respiratory diseases are stronger, thus reducing the period of infectiousness?

Secondly, opportunities for infection, $O$, are related to human behaviours modulating the magnitude and direction of contacts. For example, during winter, people cluster indoors, thus increasing the chance of contacts 8 . But indoor clustering might also occur under warm and wet conditions. Although climate affects human behaviour, including mobility, it is unlikely that a unique relationship would emerge worldwide.

Thirdly, transmission probability, $T$, is strongly affected by protective measures (for example, wearing masks, gloves and cleaning hands). However, if climate affects the viability of the virus outside the human body, it could affect transmission by contact with surfaces and aerosols'. In such cases, $T$ could vary across climatic gradients.

Finally, susceptibility to the virus, $S$, is directly linked to the immune response of the host. While it is unclear whether geographical clustering of immune responses to SARS-CoV-2 exists, it is foreseeable that they could be affected by climate $^{10}$. Such a response would affect $D$ as well as $S$.

The above discussion illustrates possible connections between SARS-CoV-2 and climate. Statistical relationships between SARS-CoV-2 and predictor variables of interest will stabilize as more data become available. So far, analyses cover the initial phases of the pandemic and there is substantial noise in key parameters. For example, during April-May 2020 the United States had the highest world COVID-19 mortality and Brazil the highest mortality in South America. This was likely related to the type of non-pharmaceutical responses to the disease (terms $O$ and $T$ in the DOTS equation). Nevertheless, signals emerge regionally and globally such that environmental factors could be linked to spread of SARS-CoV-2 and the severity of its effects. Addressing these questions will require continuous research.

\section{Conclusions}

While correlative models can provide insight concerning the environmental persistence of the pathogen (thus affecting spread of the disease), mechanistic approaches allow projecting numbers of infections and fatalities as a function of management policies. Rather than building walls across scientific disciplines, building bridges will be more effective to understand the spread of SARS-CoV-2 and its effects on human health.

Miguel B. Araújo (iD) 1,2凶, Frederico Mestre (D)2 and Babak Naimi (D) 3 ${ }^{1}$ Department of Biogeography and Global Change, National Museum of Natural Sciences, CSIC, Madrid, Spain. ${ }^{2}$ Rui Nabeiro' Biodiversity Chair, MED Institute, University of Évora, Évora, Portugal. ${ }^{3}$ Department of Geosciences and Geography, University of Helsinki, Helsinki, Finland.

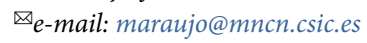

Published online: 23 June 2020 https://doi.org/10.1038/s41559-020-1246-y

References

1. Carlson, C. J., Chipperfield, J. D., Benito, B. M., Telford, R. J. \& O’Hara, R. B. Nat. Ecol. Evol. 4, 770-771 (2020). 
2. Kucharski, A. The Rules of Contagion: Why Things Spread - And Why They Stop (Profile Books, 2020).

3. Grifoni, A. et al. Cell 181, https://doi.org/10.1016/j. cell.2020.05.015 (2020)

4. Jordan, R. E. \& Adab, P. Lancet Infect. Dis. https://doi.org/10.1016/ S1473-3099(20)30395-9 (2020).
5. Araújo, M. B. \& Naimi, B. Preprint at medRxiv https://doi. org/10.1101/2020.03.12.20034728 (2020).

6. Zhu, Y., Xie, J., Huang, F. \& Cao, L. Sci. Total Environ. 727, 138704 (2020).

7. Eccles, R. Acta Oto-Laryngol. 122, 183-191 (2002).

8. Buckee, C. O., Tatem, A. J. \& Metcalf, C. J. E. Trends Parasitol. 33, 10-20 (2017).
9. van Doremalen, N. et al. N. Engl. J. Med. 382, 1564-1567 (2020) 10. Martin, L. B., Weil, Z. M. \& Nelson, R. J. Philos. Trans. R. Soc. B 363, 321-339 (2008).

Competing interests

The authors declare no competing interests. 